\title{
El concepto de racionalidad en los estudios sociales agrarios
}

\section{Gabriel Liceaga}

(iD) https://orcid.org/0000-0003-4253-0539

CONICET / Universidad Nacional de Cuyo (Argentina)

gabriel.liceaga@gmail.com

RESUMEN

En este artículo se problematiza el concepto de racionalidad, mostrando una vía posible para su utilización en el marco de los estudios sociales agrarios y la antropología económica. En primer lugar, se despeja teóricamente el camino, relacionando y distinguiendo simultáneamente dos conceptos: el de «racionalidad moderna» y el de «racionalidad económica». Luego, se exponen algunas mediaciones teórico-metodológicas que permiten realizar un uso categorial de aquellos conceptos. Más adelante, se elaboran algunas reflexiones epistemológicas sobre esta cuestión. Finalmente - y a modo de conclusión - se recapitulan las principales contribuciones realizadas, enfatizando la vinculación entre preocupaciones de orden general, ligadas al desarrollo de la modernidad, con algunas de sus plasmaciones históricas en el orden agrario y la antropología económica. Palabras clave: racionalidad, campesinado, economía campesina, comunidad, necesidades humanas. 


\section{The concept of rationality in the agrarian social studies}

\section{ABSTRACT}

In this paper we question the concept of rationality providing a possible use of it in social agrarian studies and economic anthropology. First, we theoretically clarify the path by relating and distinguishing at the same time two concepts: "modern rationality» and «economic rationality». Afterwards, we present some theoretical-methodological mediation that allows a categorical use of those concepts. Then, we elaborate some epistemological reflections on this matter. As a conclusion, we review our main contributions, emphasizing the link between general concerns, linked to the development of Modernity, with some of its historical embodiments in the agrarian order and economic anthropology.

Keywords: rationality, peasantry, peasant economics, community, human needs. 


\section{INTRODUCCIÓN}

Algunos conceptos tienen la suerte de atravesar diferentes registros teóricos, juegos de lenguaje y anclajes históricos sin perder por ello su aura de fortaleza explicativa y capacidad de persuasión. El concepto de «racionalidad» es, por larga herencia moderna, uno de ellos. No obstante, su propia ubicuidad también puede jugar malas pasadas, de modo que a veces termina abusándose del término sin que quede claro qué se quiere decir con él. En este artículo se muestra una vía posible para su utilización categorial en el marco de los estudios agrarios y la antropología económica, a propósito de referentes empíricos determinados y con un contenido conceptual explícito.

Desde luego, el interés por realizar un uso categorial del concepto de racionalidad tiene un marco de referencia que lo explica y posibilita. Existe, en efecto, una pluralidad de discursos al interior de la filosofía, las ciencias sociales y el ensayo latinoamericano que, en las últimas dos décadas, han planteado la existencia de racionalidades alternativas, que surcarían los proyectos que pugnan por construir la realidad social. Se destacan, en este sentido, autores como Boaventura de Sousa Santos (2006) y su conocida propuesta de una «ecología de saberes» destinada a poner en diálogo intercultural la pluralidad de experiencias que producen los pueblos, excedentes respecto de una forma de racionalidad occidental postulada como hegemónica. En un sentido similar, Santiago Castro Gómez y Ramón Grosfoguel (2007) propugnan la crítica de las formas eurocéntricas de conocimiento, desde el punto de vista de las racionalidades que se gestan en los márgenes del sistema moderno-colonial-capitalista. Silvia Rivera Cusicanqui (2010) contrapone la racionalidad del ayllu andino a la racionalidad estatal, como formas societales-político-económicas enfrentadas. José Quintero Weir (2011), por su parte, desarrolla interesantes reflexiones tendientes a deconstruir el modelo científico occidental, en beneficio de un «camino del saber» enraizado territorial y comunitariamente. En resumen, desde diferentes perspectivas y con disímiles niveles de profundidad, autores a los que cabe enmarcar dentro del «giro decolonial» han mostrado la vinculación íntima entre las estructuras de dominación 
coloniales en América Latina y las formas de construcción del conocimiento, problematizando así la noción de racionalidad.

Ya en el contexto de los estudios rurales y ambientales, la existencia de racionalidades enfrentadas ha sido postulada por autores como Enrique Leff (2004) y Carlos Walter Porto Goncalves (2006). En sintonía con estos planteos teóricos y con un posicionamiento crítico respecto de las consecuencias del desarrollo del agronegocio en Argentina, Diego Domínguez y Pablo Sabatino (2008) y Pablo Barbetta (2012), entre otros y otras, han conceptualizado la reemergencia de organizaciones campesinas e indígenas como índice de una disputa sobre el sentido de la tierra y la vida en el campo. En esta línea, Horacio Machado-Aráoz y Federico Paz (2016) postulan la existencia de racionalidades imbricadas, entre la «re-existencia» campesina y la lógica mercantil-capitalista.

En definitiva, la cuestión de las racionalidades (superpuestas, enfrentadas, conflictivas, imbricadas) reaparece en diversos textos críticos de las formas hegemónicas de expansión del capital, particularmente en el ámbito agrario, a veces como metaconcepto que permite englobar diferentes aspectos de la vida social y en otras ocasiones como metáfora.

De esta ambigüedad en la noción de racionalidad proviene la primera pregunta que orienta este artículo: ¿de qué contenido teórico se puede dotar a este concepto, que permita arrojar luz sobre determinados aspectos de un problema de investigación, ubicado particularmente en el ámbito de los estudios sociales agrarios? Este interrogante se agudiza al considerar que la idea de racionalidad suele ser parte del lenguaje habitual de técnicos y extensionistas (Torres, 2010) - e incluso de agricultores - y que en tales casos está asociada a criterios de eficiencia en el uso de los recursos o de maximización de las utilidades. Utilizar el término de un modo teóricamente cuidadoso se vuelve así complicado y entraña sortear diversas y entrelazadas ambigüedades.

Esta última consideración conduce a un segundo interrogante: ¿qué tratamiento del concepto de racionalidad puede realizarse, a fines de deslindar los significantes que emergen del lenguaje vulgar, con aquellos que es posible controlar teóricamente, o cuanto menos, de reconocer las continuidades y tensiones entre unos y otros? Esto parece imprescindible, en la medida en que al hablar sin más de «racionalidad» se corre el riesgo de deslizar una serie de implicaciones diversas y contradictorias.

En este artículo se intenta responder ambas preguntas a partir de una investigación doctoral en la que, por razones que se mencionarán más adelante, el concepto de racionalidad tuvo un importante papel. Para ello, en primer lugar, se 
despeja teóricamente el camino, relacionando y distinguiendo simultáneamente dos conceptos: el de «racionalidad moderna» y el de «racionalidad económica». Luego, se exponen algunas mediaciones teórico-metodológicas posibles que, en aquel marco, permitieron realizar un uso categorial del concepto de racionalidad. Más adelante, se elaboran algunas reflexiones sobre la naturaleza epistemológica del concepto de racionalidad utilizado. Finalmente, y a modo de conclusión, se recapitulan las principales contribuciones realizadas en aras de un uso consistente del concepto de racionalidad, en el marco de los estudios sociales agrarios y retomando aportes de la antropología económica.

\section{DE LA CRÍTICA DE LA RACIONALIDAD MODERNA A LA RACIONALIDAD ECONÓMICA}

El término «racionalidad» proviene del latín rationalitas, que a su vez está emparentado con la palabra ratio. Este último término significa «cálculo»o «medida», pero también, en un sentido más amplio, mienta la capacidad de juzgar, la inteligencia o la explicación de una cosa. Desde sus orígenes, por lo tanto, el concepto de racionalidad enlaza dos elementos compatibles, pero no homogéneos: la aproximación cuantitativa hacia lo real, que intenta captar matemáticamente un determinado aspecto de ella, con la facultad humana de aprehender la esencia lógica o espiritual de las cosas.

Con esta primigenia ambigüedad, el término «racionalidad» reaparece en diversas lenguas europeas, sintetizando en el lenguaje corriente lo que sería objeto de importantes discusiones filosóficas, por lo menos desde comienzos del siglo XIX: la diferencia entre la razón en general y las formas en que esta razón se cristaliza históricamente en la modernidad. En efecto, desde fines del siglo XIX, y ya con total claridad en la primera mitad del siglo XX (por ejemplo, en el rastro de Heidegger, la escuela de Frankfurt, la hermenéutica de Gadamer), muchas y variadas serán las críticas al devenir y a los efectos civilizatorios de la Ilustración y sus efectos racionalizantes.

De aquí que, desde comienzos del siglo XX, se haya utilizado la expresión «racionalidad moderna» para referir a disposiciones de la mentalidad humana alentadas por las estructuras científicas y económicas que se desarrollan y culminan en la modernidad. Esta utilización suele darse al interior de planteos que no se limitan a dimensiones estrictamente lógicas, sino que se expresan acerca de procesos ocurridos en el plano social e histórico. 
Sin duda uno de las elaboraciones teóricas más influyentes a este respecto aparece en la obra de Max Weber, en relación con el proceso de racionalización y «desencantamiento» del mundo, que caracterizaría a la época moderna (Weber, 2009). Este autor, como es sabido, concibe a la «acción racional con arreglo a fines» como aquella acción orientada por el fin, medios y consecuencias implicadas en ella, para cuya consecución se sopesan cuidadosamente los medios con los fines (Weber, 2003). Ahora bien, como ha observado Franz Hinkelammert (2003), los fines en los que piensa Weber tienden a ser parciales o limitados, con lo cual este tipo de acción desemboca en un mero cálculo (en última instancia, económico) de medios y fines. De esta manera, se piensa a la racionalidad independientemente de las consecuencias, la bondad, la justicia o belleza de los fines implicados en la acción. El proceso de racionalización subsiguiente - expresado en el plano económico, político, e incluso de las subjetividades - sería la plasmación histórica de esta forma de racionalidad, dominante durante la modernidad.

Resumiendo, existen fundadas razones para tomar como referencia teórica de la «racionalidad moderna» al concepto weberiano de «acción racional con arreglo a fines» (Weber, 2003) o al de «racionalidad medio-fin» (Hinkelammert, 2003). Esto no implica, claro está, asumir las implicaciones valorativas que Weber otorga al proceso de racionalización, ni tampoco descartar la multiplicidad y variedad de las críticas que este ha despertado.

Estos señalamientos, aunque fundamentales para avanzar en una utilización algo más que metafórica del concepto de racionalidad, no bastan para responder la primera pregunta formulada en la introducción de este artículo; a saber, de qué contenido teórico se puede dotar al concepto de racionalidad que permita su utilización en el ámbito de los estudios sociales agrarios. Debe determinarse con mayor precisión el espectro de discusiones en el que se inserta el concepto, para lo cual autores y autoras clásicos de la antropología ligados a los estudios sobre el campesinado, como Alexander Chayanov y Maurice Godelier, realizan contribuciones insoslayables a las que cabe agregar, en el ámbito latinoamericano, a Eduardo Archetti y Kristi Stölen.

Se trata, entonces, de definir el concepto de racionalidad de manera tal que permita caracterizar fenómenos empíricos ligados a las prácticas económicas de los sujetos agrarios, reconociendo que «no existe [...] racionalidad económica en sí, ni forma definitiva de racionalidad económica» (Godelier, 1974, p. 287). Para ello, es necesario también asumir que los fenómenos económicos no están separados en ningún sentido del resto de las dimensiones sociales (Comas d'Argemir, 1998), aunque estos se funden en las «relaciones sociales que participan en la 
producción y reproducción de la vida material, a través de la interacción organizada de los seres humanos y la naturaleza» (Narotzky, 2004, p. 21).

En definitiva, la utilización categorial del concepto de racionalidad en el marco de estudios agrarios o campesinos implica introducir preocupaciones filosóficas y sociales de un espectro muy general (la «racionalidad moderna» y sus plasmaciones históricas) dentro de un ámbito de discusiones más acotado, influido, en buena medida, por un enfoque sustantivista de la economía sensible al entramado de referencias que hacen parte de lo económico en cuanto «proceso institucionalizado» (Polanyi, 1974). En este ámbito existe un consolidado campo de categorías que permiten trasladar aquellas preocupaciones a los variopintos mundos agrarios, entre las cuales se destaca el concepto de racionalidad económica, según ha sido trabajado, principalmente, en el campo de la antropología económica.

\section{CONTEXTUALIZACIÓN DEL PLANTEO}

Como se señaló en la introducción, la ocasión para estas reflexiones surge del desarrollo de una investigación doctoral. En esta se analizaron determinados aspectos vinculados con un conflicto territorial que tiene lugar, desde 2006 a la fecha, en las cercanías del arroyo Los Leones (departamento de San Rafael, provincia de Mendoza, Argentina). Este conflicto tiene como protagonistas a una comunidad campesina (que habita en Los Leones) y a un grupo de profesionales del derecho, que reclama haber comprado de buena fe una parte considerable de las tierras en las que aquellos viven y trabajan. En aquella investigación se dio cuenta, particularmente, del proceso organizativo desarrollado por las familias campesinas, sus condicionamientos históricos, jurídicos y económicos, el impacto del conflicto sobre las prácticas de reproducción social (Liceaga, 2017).

En el marco de la mencionada tesis doctoral, el interés por la cuestión de la «racionalidad» surgió tempranamente. En efecto, al comenzar el trabajo de campo, uno de los abogados que disputa una fracción de tierra con la comunidad campesina realizó una interesante caracterización de su actividad productiva, que estaría signada, según su parecer, por la ausencia de «racionalidad» en el «manejo» del campo. Desacreditando el rol económico de los campesinos y campesinas - y especialmente su carácter de productores y productoras-, aquel abogado señalaba: 
El puestero ${ }^{1}$ es un tipo vago, recibe lo que le da la naturaleza. No tiene horario, jornada laboral, obligaciones, no paga impuestos. No es un contribuyente para el Estado, solo recibe. No mejora sus condiciones económico-sociales, salvo que se lo regalen. No es un agente económico en una economía regional, no emplea gente, no es un actor. No entra en el circuito económico (Ricardo, comunicación personal, agosto de 2012).

Este mismo entrevistado cuestionaba también la posibilidad de llevar adelante una actividad productiva racional en un campo sin divisiones:

Es una aberración en materia de producción [...] No se respetan los ciclos de la naturaleza (semilla, floración). Hay mucha competencia por la deforestación, el animal no respeta nada (Ricardo, comunicación personal, agosto de 2012).

Si bien esta opinión estaba expresada con cierta brusquedad, la indagación bibliográfica posterior habría de demostrar que no carecía completamente de respaldo experto. En efecto, es habitual entre técnicos y extensionistas afirmar que los puesteros y puesteras llevan adelante prácticas de manejo ganadero inadecuadas para su entorno y su bienestar económico, caracterizadas por la existencia de altos rendimientos estacionales, bajos ingresos y un fuerte impacto ambiental producto del sobrepastoreo (Torres, 2010). Como señala esta autora, estos presupuestos suelen coincidir de una u otra manera en un diagnóstico negativo respecto de la racionalidad económica de aquella población, que sería resistente al cambio, individualista y que tendría un bajo nivel educativo.

La cuestión de la racionalidad de los puesteros y puesteras apareció entonces entre las inquietudes de los propios involucrados en el conflicto de Los Leones. Esto despertó naturalmente preguntas acerca de qué debería entenderse por «racionalidad económica» o «productiva»: ¿solo existe una forma de racionalidad o podría haber una diversidad de prácticas que pudieran ser juzgadas como racionales? ¿Cuál sería un criterio apropiado para definir si la producción en Los Leones es o no racional? A partir de estos interrogantes se estructuró la construcción de un concepto de racionalidad aplicable al estudio de las prácticas económicas de la población de Los Leones.

1 En este artículo, por razones que se aclaran más adelante se ha optado por caracterizar a los pobladores y pobladoras de Los Leones como «campesinos». Genéricamente, sin embargo, no es incorrecto denominarlos «puesteros», apelativo bajo el cual ellos mismos se reconocen. El término «puestero» alude a los habitantes de los puestos, viviendas típicas de los entornos áridos y semiáridos no irrigados de la República Argentina. Un puesto, en sentido general, es una casa - típicamente de adobe o de ladrillos - en cuyas inmediaciones hay corrales y otras instalaciones ligadas a las prácticas pecuarias. Por lo general, la accesibilidad al agua orienta y estructura el asentamiento del puesto, los pozos, tanques, cisternas, acequias o aguadas (Pastor, 2005). 


\section{RACIONALIDAD Y TERRITORIALIDAD CAMPESINA}

Asumiendo, entonces, que no existe una forma de racionalidad económica, sino varias, y que esta debe ser reconstruida desde adentro, es decir, conociendo los objetivos, condicionamientos y demás aspectos de la praxis cultural de los sujetos, se describió y analizó la actividad económica en Los Leones. Por «prácticas económicas» se entendió aquellas orientadas hacia la producción de los «bienes materiales y servicios que hacen la vida posible], fundadas en las «relaciones sociales que participan en la producción y reproducción de la vida material» (Narotzky, 2004, p. 21)

Se realizó así un análisis de las principales prácticas económicas de los pobladores de Los Leones, tomando como unidad de análisis a los catorce «puestos» que se reconocen a sí mismos como parte de una «comunidad». Los puestos fueron entendidos como unidades domésticas de producción y consumo, retomándose así una tradición campesinista, que partiendo de Chayanov (1974), es continuada por autores y autoras como Archetti y Stölen (1975) y Gabriela Schiavoni (1995) en el ámbito nacional, y retomada por Laura Torres (2010) en relación con las tierras secas de la provincia de Mendoza. Se realizaron, asimismo, censos de población y animales en diferentes épocas del año, vinculando el conocimiento de las principales prácticas productivas (la cría de caprinos, bovinos, ovinos y equinos) con las reglas de herencia, las dinámicas migratorias y sus condicionamientos jurídicos, históricos, comerciales y ambientales (Liceaga, 2017).

Como síntesis de este análisis, puede reseñarse que en el territorio de Los Leones habitan alrededor de cincuenta personas agrupadas en catorce unidades domésticas. Estas se distribuyen en una franja de entre $10 \mathrm{~km}$ de ancho y $35 \mathrm{~km}$ de largo, vertebrada por un arroyo que da nombre al paraje. Las precipitaciones son escasas y variables: fluctúan entre los 200 y los 400 milímetros anuales (Gil, 2005). Además del agua del arroyo, de caudal escaso e irregular, los pobladores solo cuentan con aguadas dispersas y cambiantes y algunos reservorios generados por el trabajo humano. La disponibilidad de forraje, en consecuencia, depende exclusivamente de las lluvias y de cómo estas alimenten a las vertientes y espacios de almacenamiento, principalmente «ramblones $\rangle^{2} \mathrm{y}\langle\text { pozos balde }\rangle^{3}$.

2 Los ramblones son pequeñas lagunas que se generan a partir del agua de lluvia sobre depresiones naturales de poca profundidad. En Los Leones eventualmente son abastecidos por mangueras que derivan agua proveniente de vertientes.

3 Los pozos balde son excavaciones circulares de 1 metro de diámetro, aproximadamente, a cuyo contenido se accede mediante baldes. 


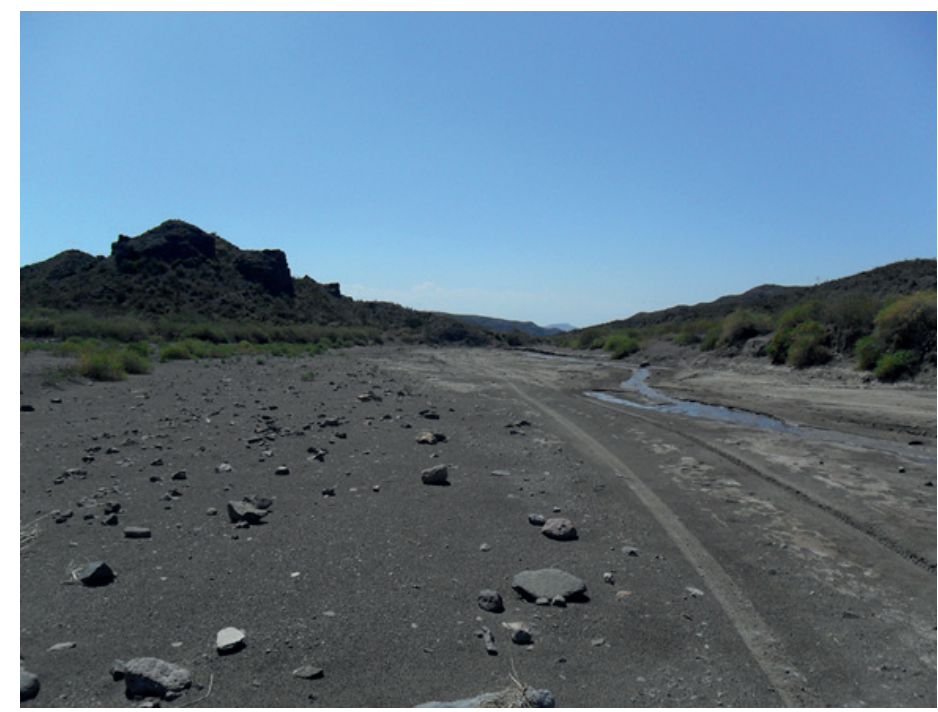

Imagen 1. Arroyo «Los Leones».

I

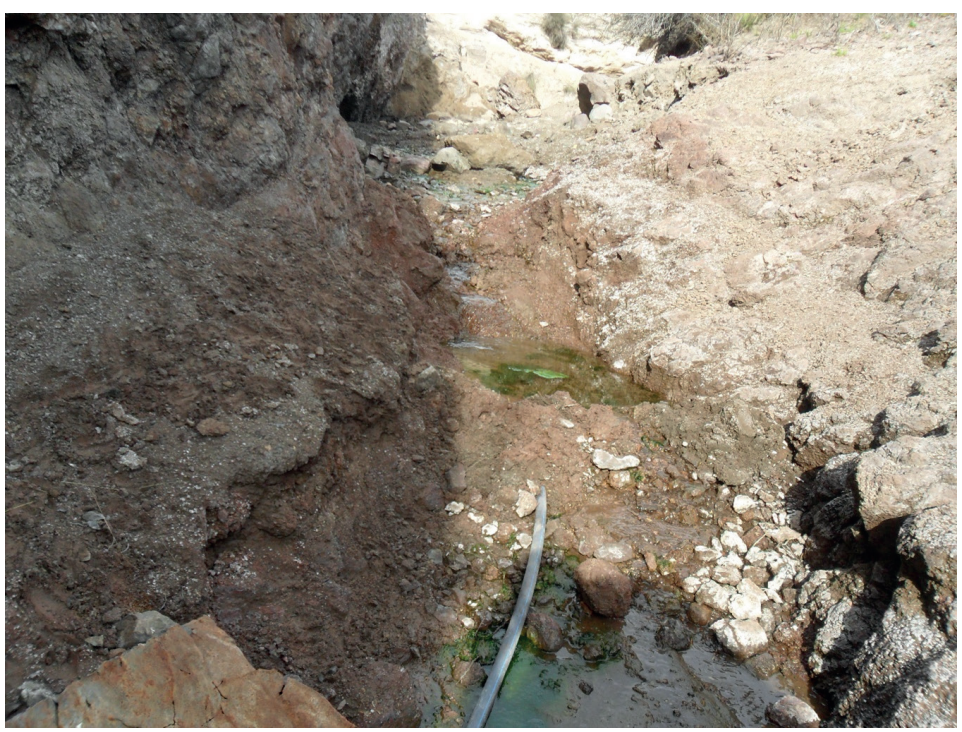

Imagen 2. Vertiente y manguera. 


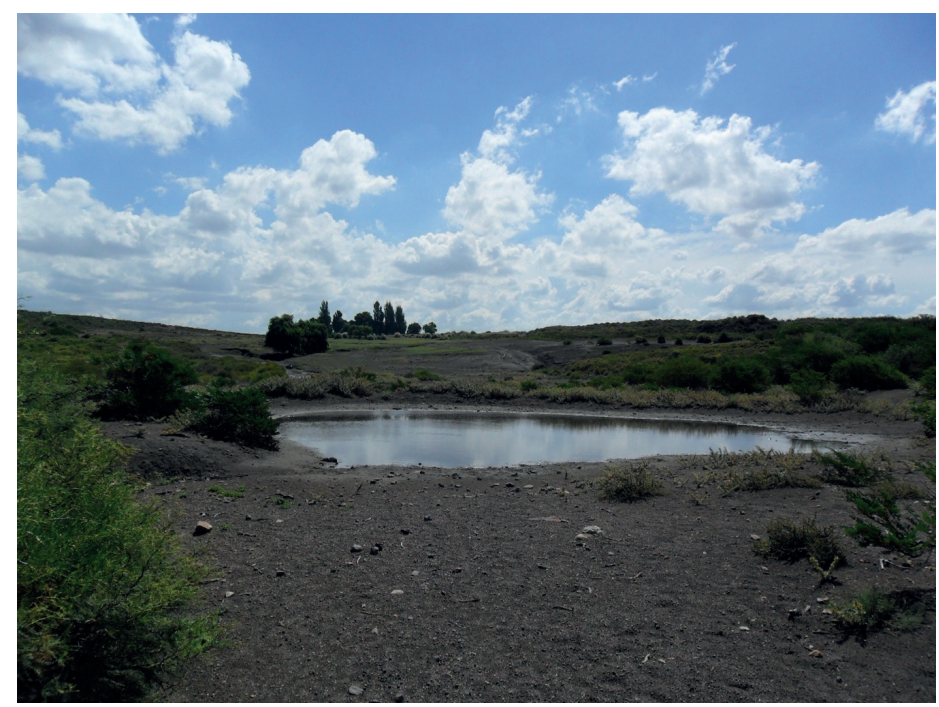

Imagen 3. Ramblón.

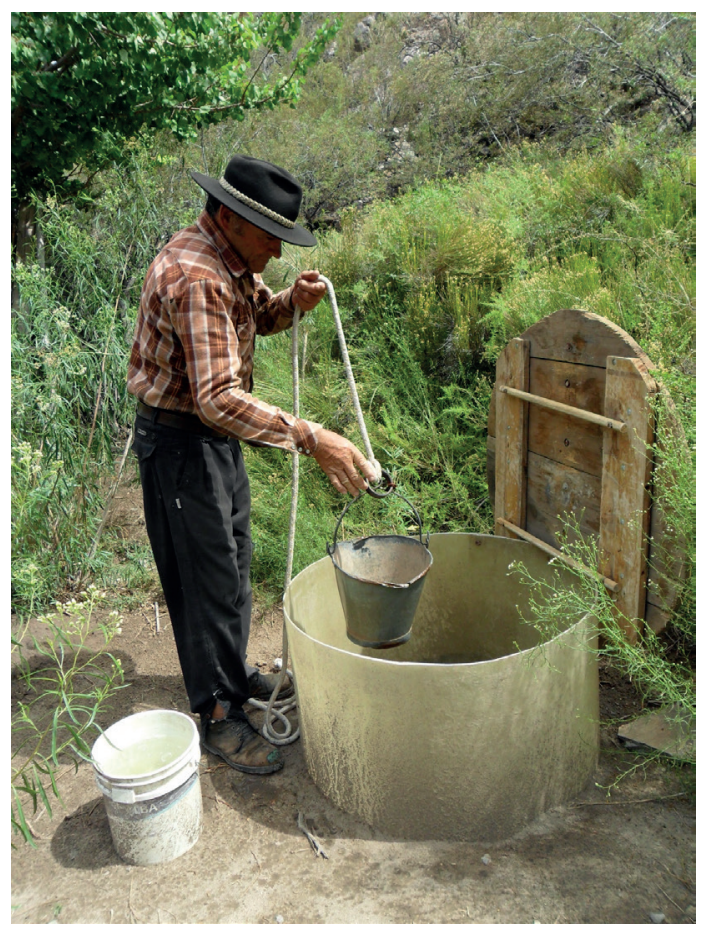

Imagen 4. Pozo balde. 
Como es común en otras zonas áridas y semiáridas del territorio argentino, los pobladores de Los Leones practican la ganadería extensiva sin utilización de cierres perimetrales. El producto principal destinado al mercado es el cabrito lechal o «chivito» (que es sacrificado y vendido cuando cuenta con sesenta días aproximadamente), seguido por los ovinos o los bovinos, dependiendo de la unidad doméstica en cuestión. Los caballos aparecen en último puesto.

Tabla 1. Composición de los planteles por unidad doméstica (2013 y 2016)

\begin{tabular}{|c|c|c|c|c|c|c|c|c|}
\hline \multirow{2}{*}{$\begin{array}{c}\text { Años } \\
\text { Unidad } \\
\text { doméstica }\end{array}$} & \multicolumn{4}{|c|}{ Año 2013} & \multicolumn{4}{|c|}{ Año 2016} \\
\hline & $\begin{array}{c}\text { Caprinos } \\
\left(\mathrm{n}^{\circ}\right)\end{array}$ & $\begin{array}{l}\text { Ovinos } \\
\left(\mathrm{n}^{\circ}\right)\end{array}$ & $\begin{array}{l}\text { Equinos } \\
\left(\mathrm{n}^{\circ}\right)\end{array}$ & $\begin{array}{c}\text { Bovinos } \\
\left(\mathrm{n}^{\circ}\right)\end{array}$ & $\begin{array}{c}\text { Caprinos } \\
\left(\mathrm{n}^{\circ}\right)\end{array}$ & $\begin{array}{c}\text { Ovinos } \\
\left(\mathrm{n}^{\circ}\right)\end{array}$ & $\begin{array}{l}\text { Equinos } \\
\left(\mathrm{n}^{\circ}\right)\end{array}$ & $\begin{array}{c}\text { Bovinos } \\
\left(\mathrm{n}^{\circ}\right)\end{array}$ \\
\hline Puesto 1 & 200 & 10 & 15 & 30 & 220 & 20 & 15 & 47 \\
\hline Puesto 2 & 180 & 30 & 20 & 50 & 250 & 40 & 10 & 60 \\
\hline Puesto 3 & 120 & 100 & 20 & 30 & 10 & 20 & 8 & 80 \\
\hline Puesto 4 & 350 & 300 & 20 & 80 & 450 & 320 & 50 & 80 \\
\hline Puesto 5 & 210 & 10 & $\mathrm{~s} / \mathrm{d}$ & $\mathrm{s} / \mathrm{d}$ & 190 & 0 & 9 & 36 \\
\hline Puesto 6 & 250 & 20 & 10 & $\mathrm{~s} / \mathrm{d}$ & 300 & 0 & 15 & 60 \\
\hline Puesto 7 & $\mathrm{~s} / \mathrm{d}$ & $\mathrm{s} / \mathrm{d}$ & $\mathrm{s} / \mathrm{d}$ & $\mathrm{s} / \mathrm{d}$ & 250 & 300 & 40 & 60 \\
\hline Puesto 8 & 200 & 150 & 30 & 20 & 175 & 10 & 20 & 20 \\
\hline Puesto 9 & 320 & 50 & 35 & 50 & 280 & 50 & 30 & 90 \\
\hline Puesto 10 & 165 & 45 & 10 & 0 & 200 & 0 & 12 & 18 \\
\hline Puesto 11 & 200 & 0 & $\mathrm{~s} / \mathrm{d}$ & 30 & 250 & 0 & 5 & 40 \\
\hline Puesto 12 & 150 & $\mathrm{~s} / \mathrm{d}$ & 5 & 10 & 200 & $\mathrm{~s} / \mathrm{d}$ & 5 & 3 \\
\hline Puesto 13 & 240 & 12 & $\mathrm{~s} / \mathrm{d}$ & 0 & 300 & 0 & 40 & 8 \\
\hline Puesto 14 & 250 & 50 & 12 & 8 & 300 & 80 & 30 & 20 \\
\hline Totales & 2765 & 777 & 177 & 308 & 3375 & 840 & 289 & 622 \\
\hline
\end{tabular}

Elaboración propia (2013 y 2016). 
El promedio de cabezas de ganado caprinos por unidad doméstica en 2013 y 2016 era de 210 y 241 animales, respectivamente. Tomando como base estos números y teniendo en cuenta las respectivas medianas (200 en 2013 y 250 en 2016) puede considerarse que el tamaño de los rodeos caprinos por unidad doméstica gira en torno a los 225 animales. Este número es superior a la media nacional, que era de cerca de 87 animales por explotación en el año 2002 (Censo Nacional Agropecuario, 2002), y poco inferior a la media provincial (Mendoza) y departamental (San Rafael) en 2008 (276 y 255 animales, respectivamente, de acuerdo con el Censo Nacional Agropecuario 2008).

La cría y venta de animales es complementada con una diversidad importante de actividades prediales (trabajo en huertas, artesanías, producción de quesos y chacinados, tejidos, entre otros) y no prediales (empleos temporarios en actividad agropecuarias y en industrias cercanas). Sobresale también una fuerte presencia de relaciones de «reciprocidad generalizada» (Sahlins, 1983) en la satisfacción de variadas necesidades, que se expresa tanto en los vínculos al interior de las unidades domésticas ${ }^{4}$, como de manera horizontal (entre diferentes unidades). Estas últimas se expresan principalmente en el transporte hacia el poblado más cercano, en las marcas y rastreo de animales y en la caza de animales predadores. Cabe señalar también que algunos miembros de las unidades domésticas suelen desarrollar prácticas migratorias transitorias (sobre todo para emplearse en actividades no prediales) o permanentes (por casamiento, estudio $\mathrm{u}$ otras razones). En este último caso suelen mantener un vínculo económico con su unidad de origen, donde conservan animales que conforman el rodeo común, pero cuya propiedad es diferenciada.

No puede abundarse aquí sobre esta cuestión, pero cabe señalar que las relaciones de reciprocidad al interior de las unidades domésticas se relacionan centralmente con los sistemas de herencia (Wolf, 1971), así como también con cuestiones de género. Quienes heredan los puestos suelen ser varones, quienes, llegado el momento, ayudan también a sus padres y madres, cuando estos no pueden hacerse cargo del grueso de las tareas productivas. 


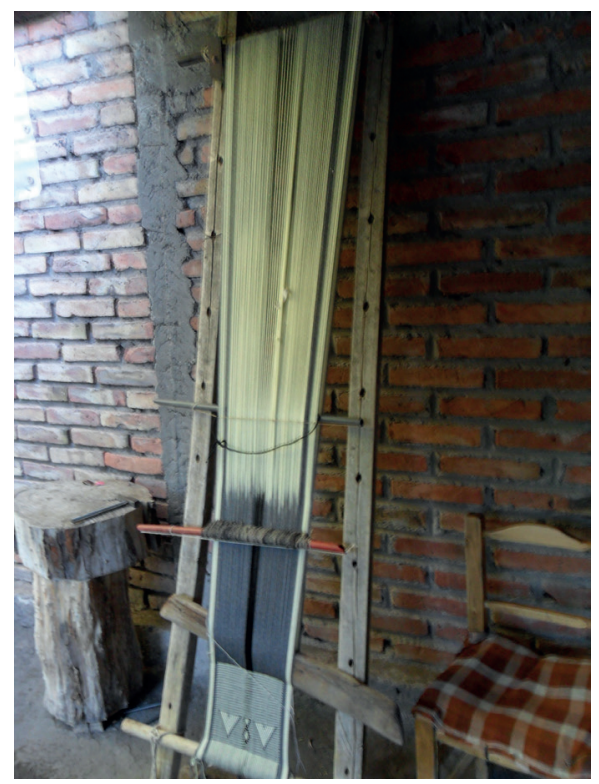

Imagen 5. Tejido tradicional.

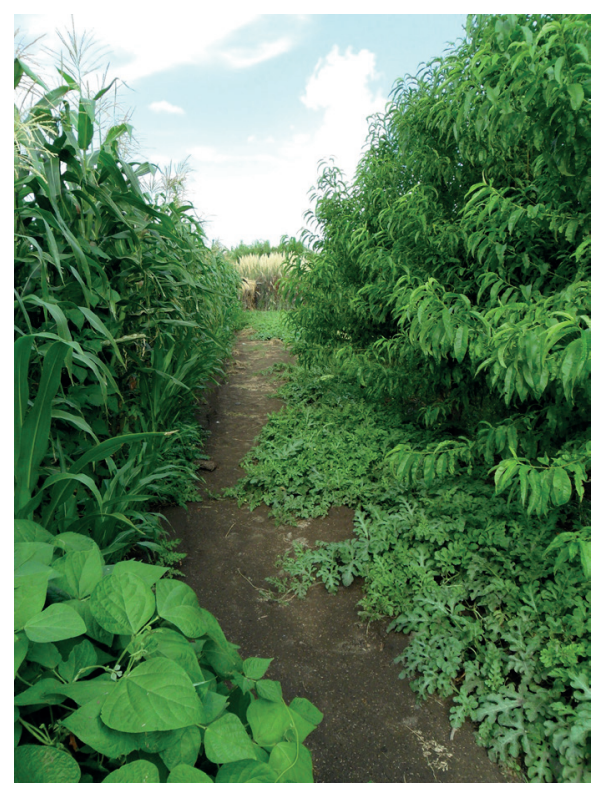

Imagen 6. Huerta. 
En conjunto, puede afirmarse que las prácticas económicas de los campesinos y campesinas de Los Leones se encuadran dentro de los criterios que Sahlins (1983) propone como característicos de las «economías domésticas» y Van der Ploeg (2008) de las «economías campesinas» (trabajo familiar o comunitario, el control de la tierra y la orientación de la producción hacia la reproducción de la unidad doméstica). En el plano nacional otros autores agregan a estas características las dificultades estructurales para la acumulación, el control formal del proceso productivo, el origen agropecuario de una parte importante de los ingresos, la escasa disponibilidad de recursos tecnológicos y la subordinación a la mayoría de los actores con las que interactúan (Hocsman, 2003; Cáceres y otros, 2006).

De esta forma, los campesinos y campesinas de Los Leones y el territorio construido por esta colectividad pueden ser caracterizados como «campesinos». Ahora bien, si los pobladores de Los Leones desarrollan una territorialidad campesina, ¿qué orden subyace a este modo de territorialización? En otras palabras, ¿qué caracteriza a la racionalidad económica campesina?

\section{Racionalidad económica campesina}

En la búsqueda de referencias teóricas para la caracterización de una racionalidad económica campesina sobresale la figura de Chayanov. Uno de sus aportes teóricos fundamentales se sintetiza en lo que comúnmente se denomina ley de Chayanov, que puede formularse de la siguiente manera:

Puesto que el estímulo básico de la familia trabajadora para la actividad económica es la necesidad de satisfacer las demandas de sus consumidores y dado que sus manos son el medio principal para ello, debemos esperar, ante todo, que el volumen de la actividad económica de la familia corresponda cuantitativamente en forma aproximada a estos elementos básicos en la composición familiar (Chayanov, 1974, p. 56).

A partir de este pasaje pueden emprenderse diversas reflexiones teóricas, pero el dato esencial por ahora es el siguiente: las unidades campesinas persiguen

5 En la cita precedente queda también implícita la idea de que habría cierto equilibrio entre el volumen de actividad económica (o grado de «autoexplotación» del trabajo campesino), el tamaño y ciclo vital de las familias y sus demandas de consumo. A partir de estos elementos, Chayanov (1974) saca la conclusión de que el grado de «autoexplotación campesina» sigue una curva decreciente en relación con la cantidad de bienes que produce su trabajo y el grado de satisfacción de necesidades que este le reporta. Theodor Shanin conecta esta cuestión con la noción de «óptimos diferenciales», es decir, con la idea de que «en regiones y subactividades 
como fin la satisfacción de las necesidades. Los campesinos se preocupan por su productividad y por el precio de sus productos, por supuesto, pero esto en función de la posibilidad de perseverar en la producción y consolidarse en el territorio, y no - retomando la expresión marxiana - atendiendo a la «valorización del valor».

A esta caracterización se agregó otro rasgo que Raúl Prada (2014) denomina la «matriz comunitaria» de las formaciones campesinas:

No hay una oikonomía campesina, sino una pluralidad de formas de oikonomía campesina. Precisamente esta pluralidad de formas se debe a su racionalidad combinatoria; por lo tanto, el perfil adquirido en un ecosistema, en una geografía local, en ámbito regional, dependen de la composición de la combinatoria. Lo sugerente de la oikonomía campesina es que logra preservar el tejido comunitario en la amalgama de sus combinaciones y composiciones $[\ldots]$ En la medida que esta amalgama de relaciones y combinaciones de distintas lógicas, que obviamente, terminan internalizándose, no afecte la reproducción del tejido comunitario, la racionalidad combinatoria es parte de las estrategias de sobrevivencia y reproducción de la comunidad (Prada, 2014, pp. 124-125).

Siguiendo a Prada, por lo tanto, la recreación de la comunidad es tan esencial para la constitución de una economía campesina, como la producción de alimentos y la posesión de los medios de producción. La «racionalidad combinatoria» a la que alude puede transformarse, pero siempre en función de la reproducción del conjunto.

Se destacan, por lo tanto, dos dimensiones fundamentales del concepto de racionalidad económica campesina: en primer lugar, la satisfacción de las necesidades; en segundo lugar, la recreación del tejido comunitario. A continuación se plantea una manera de abordar estas categorías en un plano empírico, a propósito del caso particular de Los Leones

agrarias diferentes a cualquier nivel tecnológico dado, hay tamaños óptimos de empresas diferentes y que sus variaciones tanto al alza como a la baja harán que su productividad decaiga» (Shanin, 1988, p. 148). Luego, para comprender la lógica productiva campesina, habría que considerar la gravitación que pudiera tener el contexto social de la agricultura, la disponibilidad de la familia y la ayuda de vecinos, circunstancias que podrían redundar, ocasionalmente, en que las economías no capitalistas presentaran ventajas sobre las formas de producción capitalista. 
La satisfacción de necesidades como índice de la racionalidad económica campesina

Una mañana fresca de verano, un poblador de Los Leones se pasea por los alrededores de su vivienda con un sombrero en la mano. De repente encuentra lo que buscaba: un gallinero que habia perdido de vista. Ubica algunos de los huevos en la copa del sombrero y se acerca a la vivienda. Al entrar comenta, alegre: "con esto ya me hice el día». Más tarde toma un vaso de vino mezclado con jugo y, con satisfacción, dice, «no hay vino malo».

(Notas de campo, 2013)

Si las prácticas económicas en Los Leones están orientadas esencialmente a la satisfacción de las necesidades de sus pobladores, cabe preguntarse: ¿logran este objetivo? ¿Los campesinos y campesinas de Los Leones cubren sus necesidades de un modo más o menos satisfactorio? La respuesta positiva o negativa a estas preguntas constituye un índice acerca de la racionalidad económica de sus prácticas.

A fines de avanzar en esta difícil cuestión, se toma como referencia la propuesta teórica de Doyal y Gough (1994) acerca de la estructura de las necesidades humanas. Estos autores sostienen que, si bien la construcción de las necesidades es histórica, pueden establecerse metas universales y objetivas que los individuos deben lograr para optimizar sus oportunidades de vida y prevenir daños graves. Estas son básicamente dos: la sobrevivencia física y la autonomía personal.

Estas metas universales pueden ser satisfechas - en un grado variable- a través de múltiples satisfactores culturalmente determinados. Dichos satisfactores pueden ser considerados como el conjunto de bienes, servicios y relaciones que mejoran la salud física y la autonomía en todas las culturas, proveyendo así el puente entre las necesidades básicas universales y los satisfactores relativos. Puede identificarse, así, un conjunto de fines o «necesidades intermedias» que deben satisfacerse para alcanzar aquellas metas.

Las necesidades intermedias (es decir, las condiciones mínimas para la supervivencia física y el desarrollo de la autonomía personal) serían las siguientes: (i) comida nutritiva y agua limpia; (ii) vivienda protectora; (iii) ambiente de trabajo no dañino; (iv) medio ambiente no perjudicial; (v) atención sanitaria apropiada; (vi) seguridad en la infancia; (vii) relaciones primarias significativas; (viii) seguridad física; (ix) seguridad económica; (x) educación apropiada, y (xi) control de nacimientos, embarazo y parto. 
A partir del trabajo de campo desarrollado en Liceaga (2017), se contrasta este listado de necesidades con la situación de los campesinos de Los Leones. En relación con las primeras cuatro (según la clasificación de Doyal y Gough), no se verifican dificultades importantes. La disponibilidad de alimentos está asegurada, y en el trabajo de campo realizado durante el trayecto de la tesis doctoral no surgió que los campesinos y campesinas sufrieran de enfermedades que pudiesen atribuirse a una mala alimentación, a un ambiente perjudicial o a la mala calidad del agua ${ }^{6}$.

Las necesidades de «seguridad en la infancia»y «relaciones primarias significativas» — hasta donde es posible afirmar sin recurrir a tests psicológicos u otros instrumentos clínicos - también estarían garantizadas, mientras que tampoco se observaron dificultades significativas relativas a la «seguridad física» o al «control de nacimientos embarazo y parto».

Más problemática es la cuestión de la «seguridad económica». La existencia de migraciones ocasionales o cuasipermanentes, documentada durante el trabajo de campo, indica que las actividades prediales no siempre aseguran un mínimo de ingresos satisfactorio para los campesinos considerados como individuos ni para la reproducción de la unidad doméstica. Estas dificultades suelen asociarse sobre todo a variables ambientales (fundamentalmente, el estado de las pasturas, influido a su vez de manera principal por las lluvias), que influirían sobre aspectos sociales y económicos.

Por otra parte, en relación con la atención sanitaria, pueden hacerse algunas consideraciones interesantes. En el Nihuil y en Las Malvinas (los poblados más cercanos a la comunidad) existen centros de salud, es decir, de atención primaria, donde concurren los pobladores de Los Leones. Más lejos (a unos $100 \mathrm{~km}$, en la cabecera departamental) hay un hospital de alta complejidad en el que se pueden tratar enfermedades más severas, aunque algunos campesinos prefieren viajar periódicamente hasta la ciudad capital (Mendoza) para realizarse tratamientos más complejos (notas de campo, 2013).

No obstante, al margen de esta relativa accesibilidad a la salud pública, es interesante que, en el centro de salud del Nihuil, existe una placa que descalifica notablemente las prácticas medicinales tradicionales:

$6 \quad$ No obstante, respecto de este último punto conviene mantener ciertas reservas, ya que una serie de análisis físico-químicos realizados en siete puestos cercanos a Los Leones, muestran que los niveles de salinidad de las aguas utilizadas son en su mayoría entre moderados y altos (IADIZA, 2008). Esta circunstancia alerta acerca de posibles riesgos para la salud de personas y animales y alienta a la realización de estudios específicos para la zona en cuestión. 


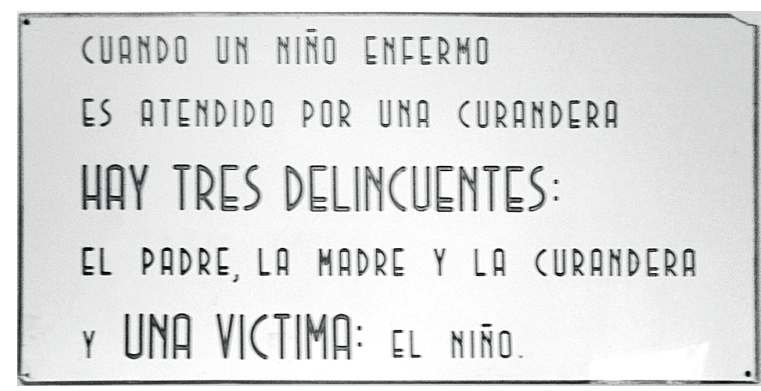

\section{Imagen 7. Cartel en centro de salud de El Nihuil (San Rafael, Mendoza).}

Llama la atención la dureza con que las prácticas tradicionales desarrolladas por «curanderas» son juzgadas y completamente desacreditadas en la institución de salud más próxima.

También a propósito de la necesidad de una «educación apropiada» pueden formularse algunas reflexiones. Al comienzo del trabajo de campo de la investigación, un entrevistado aportó interesantes puntos de vista sobre la educación de su hijo varón:

No sé hasta qué grado va a llegar a la escuela. Yo creo que con un primario. Otra de las cosas que no veo el por qué le exigen tanto a los jóvenes, por ejemplo, de que tenés que tener un primario y un secundario si sos del campo. Acá muchos de los jóvenes que se van a la ciudad se van a partir de la escuela, porque terminás un primario, después tenés un secundario que te lo exigen y de ahí como que en la escuela le cambiaran la mentalidad de que en el campo no podés vivir, si no tenés estudios no podés hacer nada, y no. Yo hice séptimo grado, tuve nada más que un primario y sin embargo, vivo bien (Ariel, julio de 2012).

En este sentido, aportaba después:

Eso es lo que veo yo en la escuela, veo que niños que estudian en la escuela, niños de campo, después terminan, terminan el estudio y no les da para más. Por ejemplo, en la escuela El Nihuil, personas que han terminado el secundario y están trabajando en una fábrica [...] Yo antes de darle un estudio, un secundario a mi hijo, me parece que le enseñaría lo que yo aprendí en el campo. Cómo criar, cómo atender un animal, si un animal también te va a dar beneficios a la larga (Ariel, julio de 2012). 
Según estas reflexiones del entrevistado - y siguiendo la terminología de Doyal y Gough (1994) — la educación al alcance de la mayoría no sería «apropiada», ya que no le brindaría conocimientos útiles para permanecer en el campo, sino que, por el contrario, les «cambiaría la mentalidad», desvalorizando las oportunidades que se abren a partir de la permanencia en el territorio y los saberes asociados con esta. En un sentido similar — aportaba otra entrevistada-: «Era como también ir a la escuela y no te incentivaba a estar en el campo, porque nunca te hablaron del campo» (Anyelén, abril de 2016). En resumen, los entrevistados y entrevistadas coinciden en que la escuela tradicional no brinda herramientas valiosas para la vida en el campo, aunque sí para la convivencia y la socialización en otros entornos. Pero difícilmente podría afirmarse que la educación sea «apropiada» para fortalecer saberes que tiendan a la permanencia en el territorio, a lo que se suma que, al no existir ofertas de educación superior a menos de 100 kilómetros, quienes desean continuar estudiando deban alejarse, planteándose así la opción entre permanecer y estudiar como una disyuntiva: «A José no le gustaba el campo, le gustaba el estudio» (notas de campo, junio de 2016).

En resumen, siguiendo la clasificación propuesta por Doyal y Gough (1994) - $\mathrm{y}$ atendiendo al trabajo de campo desarrollado - puede afirmarse que, en términos generales, los campesinos y campesinas de Los Leones resuelven satisfactoriamente sus necesidades. Existen observaciones que se pueden realizar en algunos aspectos, pero puede afirmarse que la racionalidad combinatoria (Prada, 2014) de los campesinos de la zona les permite, en términos generales, alcanzar un nivel de vida más o menos satisfactorio en términos de salud y autonomía personal.

En apoyo de esta afirmación cabe tener en cuenta algunos señalamientos que hiciera alguna entrevistada respecto de qué sería la «pobreza». Frente a las concepciones de otras personas respecto de la vida en el campo, sostenía:

Yo no sabría decir la definición de pobre [...] Yo siento que en el campo siempre nos dijeron que éramos pobres, porque el resto nos «empobrece» [...] como que los demás nos han empobrecido en lo económico. En lo económico con respecto al dinero. Podemos no tener plata, pero podemos tener otro montón de cosas que no necesitamos plata para tenerlas. El hecho de tener animales es tener una gran inversión y no todos lo ven como una gran inversión. Como que tener una inversión es tener un vehículo, tener qué sé yo, plata en el banco, cosas así. Pero si tenés animales tenés mucho invertido y ya sea, lo podés transformar en plata o lo podés tener como animales. A la hora de querer comer carne la tenés, porque no tenés que ir a comprarla (Belén, marzo de 2016). 
Aquí la entrevistada pone de manifiesto que la «pobreza» puede ser considerada de muchas maneras y que dentro de ellas juegan un papel importante aquellas de orden cultural y político. La percepción respecto de la pobreza no solo tiene que ver con los recursos de los que se dispone, sino también con la valoración que de ellos se haga. En este proceso se ponen en juego también otras dimensiones, que en términos de Doyal y Gough (1994) se expresarían en términos de la «autonomía personal» que los campesinos y campesinas — no necesaria ni automáticamente - pueden tener: «Tener tus propias cosas y no tener que estar trabajándole a otro [...] poder tener tus cosas y saber lo que comés» (Anyelén, marzo de 2016). En este último pasaje, la riqueza es asociada con los grados de autonomía que se pueda tener en la organización de las formas de trabajo, y en última instancia, con la recreación de un modo de vida.

\section{Recreación del tejido comunitario}

A partir del trabajo de campo desarrollado, resaltan dos aspectos que condicionan la recreación del tejido comunitario en Los Leones. El primero de ellos es el de la organización de la actividad ganadera a campo abierto. El segundo se refiere a lo que se caracterizará como «límites sociales a la acumulación».

Como ya se mencionó, los campesinos y campesinas de Los Leones organizan su actividad ganadera sin utilizar cierres perimetrales (alambrados), sino que lo hacen, como suele denominarse, «a campo abierto». Esta forma de organizar la pastura y cuidado de los animales no es solo el producto de una limitación económica que impediría la implementación de barreras físicas entre las majadas, sino que es una práctica desarrollada en diversas regiones del país, que posee un sentido y una explicación que se deben desentrañar. Laura Torres (2008) sintetiza esta cuestión, a propósito de los entornos no irrigados del noreste de la provincia de Mendoza. Su argumentación mutatis mutandis puede aplicarse a la realidad de Los Leones:

La dinámica de uso de los recursos, en este caso de las pasturas, se resuelve mediante una particular organización de las actividades que deriva en la inexistencia de límites al pastoreo. Los niveles de degradación que exhiben los campos harían imposible mantener en encierro las cantidades de ganado que los puesteros poseen, mientras que mantener el pastoreo a campo abierto permite que los animales se trasladen en busca de las zonas menos castigadas. Contrariamente, imponer límites a las zonas de pastoreo mediante el uso de alambrados implicaría que, tempranamente, los campos parcelados se degraden aún más [...] y 
que los animales de la explotación vean limitadas al extremo sus posibilidades de alimentación, enfrentándose a una muerte segura. El campo abierto, con aguadas compartidas, es, bajo esta perspectiva, un modo de organizar el territorio que presenta sendas ventajas respecto de las modalidades dominantes en las zonas de oasis, que indefectiblemente marcan los límites de las propiedades mediante el uso de alambrados (Torres, 2008, p. 81).

Como señala Torres, el pastoreo a campo abierto tiene un sentido no solo social sino incluso ambiental. Esto no quiere decir, por supuesto, que la ganadería llevada a cabo con esos parámetros sea invariablemente la mejor forma imaginable de gestión del territorio. El punto es que las prácticas actuales tienen sentido en las condiciones particulares en que se encuentran los campesinos de Los Leones, es decir, en un territorio extenso con aguadas dispersas y cambiantes, en el que la disponibilidad de forraje depende exclusivamente de las lluvias.

El segundo aspecto que importa a la recreación del tejido comunitario tiene que ver con los límites que existen para la cantidad de animales que tiene cada unidad doméstica. Esta cantidad influye en la calidad y cantidad del forraje al que puede aspirar el conjunto de los animales y a la posibilidad de formación de nuevos puestos; pero también, obviamente, en los procesos de diferenciación social que pudieran generarse al interior de la comunidad. ¿Cómo se regulan estos límites?

En relación con esto cabe señalar en primer lugar que no se observan - en Los Leones - procesos de diferenciación social, que influyan en las formas de manejo pecuario, la contratación de fuerza de trabajo, el acceso diferencial a redes de comercialización u otros indicadores que impliquen un cambio cualitativo en la producción (Liceaga, 2017). Esta circunstancia no resulta, por supuesto, de alguna clase de normativa o acuerdos intracomunitarios, sino que está condicionada por la disponibilidad de fuerza de trabajo de las unidades domésticas que representa, como señala Bartra (2006, p. 291), «una magnitud más o menos rígida a la que, de no existir otros factores limitantes, tenderá a adaptarse la escala de la actividad económica». Esta última varía de acuerdo con el número de sus miembros en condiciones de «atender» a los animales, es decir, de ocuparse de las tareas que se realizan en el campo, como en los alrededores de los puestos. Este máximo de fuerza de trabajo necesario para atender a los animales tiene su pico entre los meses de octubre y marzo, pero especialmente en octubre, cuando acontecen las pariciones.

Siguiendo la lógica argumental de Chayanov y adecuándola al contexto en cuestión, puede afirmarse lo siguiente: el relativo «equilibrio» en Los Leones se da entre la disponibilidad de fuerza de trabajo, el consiguiente grado de autoexplotación posible y la cantidad de animales que se puede tener de un modo 
sostenible en el tiempo. Este número de animales rondaría en torno a las doscientas cabras por grupo doméstico - tal como se manifiesta en entrevistas y en notas de campo-, pero también en el número promedio de animales por unidad doméstica, que es de alrededor de 225 animales (véase tabla 1).

Si se recuerda el concepto de óptimos diferenciales de Chayanov (1974), se encuentra entonces que el tamaño óptimo de los rodeos es de doscientos animales. Por debajo de ese número habría fuerza de trabajo subutilizada; por encima de ese número, las dificultades de manejo tienden a traducirse en una mayor mortandad de animales, ya sea en épocas de sequía o de tormentas, como consecuencia de los ataques de pumas y zorros, pérdidas u otras circunstancias. Pero, sobre todo, como afirma Belén (marzo de 2016): «No hay que vivir para los animales sino vivir de los animales».

En resumen, la cantidad de animales se estructura de acuerdo con la capacidad de trabajo, según las modalidades existentes de manejo. Esto marca un límite social para la cantidad de animales, condicionado a su vez por cuestiones ambientales. Pero este límite redunda además en un límite para la acumulación individual o familiar. Esto no quiere decir, por supuesto, que este límite social y natural para la acumulación sea algo deseado por los campesinos, ya que, como señala Luis Llambí (1981), «no debe confundirse lo que el campesino se propone con lo que logra alcanzar de hecho y muy a pesar de él» (p. 133), sino que la recreación del tejido comunitario encuentra en aquellos límites uno de sus presupuestos.

\section{ASPECTOS EPISTEMOLÓGICOS}

El desafío teórico-metodológico planteado en este artículo es el de pensar en un plano empírico un concepto sumamente abstracto, como el de racionalidad. En los apartados anteriores se mostró una vía posible para resolver esta cuestión, que responde a una situación empírica específica, sin por ello olvidar el marco general en el que se desenvuelve la indagación, esto es, la crítica de la modernidad.

Ahora bien, esta forma de tratar y construir al concepto no responde a los criterios propios del método hipotético-deductivo, ni de sus revisiones falsacionistas, así como tampoco a los métodos de «construcción de teoría» que operan con una lógica predominantemente inductiva. ¿Qué naturaleza epistemológica tiene entonces el concepto de racionalidad aquí utilizado?

La respuesta a esta pregunta se encuentra dentro de la propuesta teórico-metodológica formulada por Enrique de la Garza Toledo, denominada 
«configuracionismo latinoamericano» (de la Garza Toledo, 2011). Dentro de este planteo, que se propone reinsertar al marxismo dentro de las discusiones contemporáneas sobre el método en las ciencias sociales; las configuraciones teóricas ocupan el lugar que - dentro del positivismo - ocupaba el sistema hipotético-deductivo, en última instancia, la teoría. De la Garza Toledo propone un uso reconstructivo (no deductivo) de la teoría acumulada: no se trata ya de verificar los conceptos en el mundo empírico, sino de desarticularlos a partir de la confrontación empírica y rearticularlos en función de los objetos (descripción articulada). El trabajo conceptual no procede, por lo tanto, deductivamente desde el concepto al dato, sino que confronta las diferentes dimensiones de un concepto con los datos, generando conglomerados de indicadores que «rodean» al concepto. A partir de ello, se aceptan, desechan o se incorporan dimensiones no consideradas al principio (de la Garza Toledo, 2002).

El resultado del trabajo conceptual es, así, la construcción de una configuración, con potencialidades, limitaciones e incertidumbres a llenar por la acción de los sujetos; configuración que no rechaza de por sí las contradicciones, ambigüedades y oscuridades que, de cualquier modo, se dan en las realidades empíricas.

Construir el concepto de racionalidad en términos de una configuración permite echar luz sobre una de las preguntas que orientan este artículo, esta es, cómo deslindar el contenido teórico propuesto de la pluralidad de dimensiones de sentido que lo ligan al lenguaje corriente, o cuando menos, de controlar y reconocer estas asociaciones. Esta necesidad - omnipresente en las ciencias sociales, pero especialmente transparente al momento de utilizar conceptos como el de racionalidad - no es, claro está, novedosa ni de fácil resolución. A propósito de esta cuestión, De la Garza Toledo sostiene que, si la relación entre conceptos, indicadores y datos no es estrictamente deductiva, otras formas de mediación propias del razonamiento cotidiano pueden encontrar un lugar al interior del discurso científico. De esta manera, los razonamientos y los términos del lenguaje común pueden formar parte de la reconstrucción teórica no como «residuo», sino como parte constitutiva de lo científico (de la Garza Toledo, 2011). Las relaciones entre el razonamiento sistemático y el sentido común son fluidas; los conceptos (cuando menos algunos de ellos) son mestizos, lo que no significa recaer en una sociología espontánea (Bourdieu, 2002), sino atender al ida y vuelta entre el lenguaje común, las síntesis teóricas y la reconstrucción intencional y específica que se haga de un concepto al interior de un determinado problema de investigación, que, en todo caso, deberá ser consciente de la multiplicidad de dimensiones que lo constituyen. 
De esta manera, el concepto de racionalidad aparece como un código cultural privilegiado en su función de dar sentido, tarea que corresponde al sujeto investigador. Este debe hacerlo a partir de la construcción de una configuración que atienda a la situación concreta, valiéndose del código cultural disponible en la ciencia social y el sentido común. La noción de racionalidad puede ser pensada, así, como una «estructura cultural» (de la Garza Toledo, 2001), es decir, como un código de sentido ordenador de prácticas y discursos, donde deben verificarse y ser captadas sus diversas significaciones.

\section{CONSIDERACIONES FINALES}

Al comienzo de este artículo se planteaban dos preguntas ordenadoras: de qué contenido teórico dotar al concepto de racionalidad, que permitiera su utilización categorial en el marco de los estudios agrarios, y cómo lidiar con las confusiones y continuidades entre el lenguaje científico y el lenguaje vulgar. Es momento aquí de aclarar cuáles son las contribuciones que se realizaron en este artículo para responder a estas preguntas.

Respecto de la primera cuestión, cabe señalar que el recorrido que conecta al concepto de «racionalidad moderna» con el de «racionalidad económica» permitió vincular preocupaciones de orden general, ligadas al desarrollo de la modernidad, con algunas de sus plasmaciones históricas en el orden agrario. Este es un modo posible de volver operativo al concepto de «racionalidad» para la interpretación de realidades circunscriptas en tiempo y lugar. A la inversa, ubicar las preocupaciones de orden antropológico-económico dentro de la crítica de la modernidad permitió alzar la mirada sobre la praxis inmediata de los sujetos y enriquecer su interpretación teórica.

Asimismo, dotar de un contenido determinado al concepto de racionalidad posibilitó alumbrar algunos aspectos del problema de investigación que dio origen a las indagaciones; particularmente a cómo se configura la racionalidad económica de Los Leones, que, al igual que la de otras comunidades campesinas busca «garantizar la subsistencia y, eventualmente, una cierta condición social» (Bartra, 2006, p. 259). La condición comunitaria y la satisfacción de las necesidades se revelaron así como dos claves fundamentales de la campesineidad.

En relación con la segunda de las preguntas, acerca de las relaciones entre el lenguaje científico y el lenguaje vulgar, cabe agregar a lo dicho en el apartado anterior que la utilización consciente del concepto hizo posible dejar de lado cualquier connotación normativa que pudiera traer consigo, dimensión tácita que 
acaso constituya el peor lastre del lenguaje vulgar. Este, qué duda cabe, es un paso preliminar para cumplir la tarea del antropológo o antropóloga que, como sostuvieran Archetti y Stölen (1975, p. 16), radica en «descubrir la racionalidad propia, específica de cada sistema económico».

Para finalizar, cabe señalar que el trabajo epistemológico, teórico y metodológico efectuado no pretende ser más que una sugerencia inspiradora para tratamientos similares de este y otros conceptos, que permitan alumbrar determinados aspectos de determinadas problemáticas, sin perder de vista cómo los grandes temas de la modernidad atraviesan e influyen — se sea o no consciente de ello- sobre las elaboraciones teóricas.

\section{REFERENCIAS BIBLIOGRÁFICAS}

Archetti, Eduardo. y Stölen, Kristi (1975). Explotación familiar y acumulación de capital en el campo argentino. Buenos Aires: Siglo XXI.

Barbetta, Pablo (2012). Ecologías de los saberes campesinos: más allá del epistemicidio de la ciencia moderna: reflexiones a partir del caso del Movimiento Campesino de Santiago del Estero - Vía Campesina. Buenos Aires: CLACSO.

Bartra, Armando (2006). El capital en su laberinto. De la renta de la tierra a la renta de la vida. México, D.F.: UNAM-Ítaca.

Bourdieu, Pierre (2002). El oficio de sociólogo. Buenos Aires: Siglo XXI.

Cáceres, Daniel.; Silvetti, Felicitas.; Ferrer, Guillermo y Soto, Gustavo (2006). Y...vivimos de las cabras. Transformaciones sociales y tecnológicas de la Capricultura. Buenos Aires: La Colmena.

Castro Gómez, Santiago. y Grosfoguel, Ramón (comps.) (2007). El giro decolonial: reflexiones para una diversidad epistémica más allá del capitalismo global. Bogotá: Siglo del Hombre; Universidad Central, Instituto de Estudios Sociales Contemporáneos y Pontificia Universidad Javeriana, Instituto Pensar.

Chayanov, Aleksandr (1974). La organización de la unidad económica campesina. Buenos Aires: Nueva Visión.

Censo Nacional Agropecuario (2002). Recuperado de indec.gov.ar.

Censo Nacional Agropecuario (2008). Recuperado de indec.gov.ar.

Comas D’Argemir, Dolors (1998). Antropología económica. Barcelona: Ariel.

De la Garza Toledo, Enrique (2001). Subjetividad, cultura y estructura. Iztapalapa, UAMI, 21(50), enero-junio, 83-104. 
De la Garza Toledo, Enrique (2002). La configuración como alternativa del concepto estándar de la teoría. En Hugo Zemelman (ed.), Epistemología y sujetos (pp. 17-38). México, D.F.: UNAM-Plaza y Valdés.

De la Garza Toledo, Enrique (2011). La metodología marxista y el configuracionismo latinoamericano. En Enrique. de la Garza Toledo y Gustavo Leyva (eds.), Tratado de metodología de las ciencias sociales: perspectivas actuales (pp. 236-266). México, D.F.: Fondo de Cultura Económica.

Liceaga, Gabriel (2017). Territorialidad y resistencias campesinas: el conflicto de Los Leones (Mendoza, Argentina). Tesis doctoral.

De Sousa Santos, Boaventura (2006). Renovar la teoría crítica y reinventar la emancipación social (encuentros en Buenos Aires). Recuperado de http://bibliotecavirtual. clacso.org.ar/ar/libros/edicion/santos/Prologo.pdf

Domínguez, Diego y Sabatino, Pablo (2008). El conflicto por la tierra en la actualidad latinoamericana: del acceso a la tierra a las luchas por el territorio. Recuperado de http://bibliotecavirtual.clacso.org.ar/ar/libros/becas/2008/deuda/doming.pdf.

Gil, Adolfo (2005). Arqueología de La Payunia (Mendoza, Argentina). El poblamiento humano en los márgenes de la agricultura. Oxford: Archaeopress.

Godelier, Maurice (1974). Racionalidad e irracionalidad en economía. México, D.F.: Siglo XXI.

Doyal, Len y Gough, Ian (1994). Teoría de las necesidades humanas. Barcelona: Icaria.

Hinkelammert, Franz (2003). El sujeto y la ley. Heredia: EUNA.

Hocsman, Luis Daniel (2003). Reproducción social campesina. Tierra, trabajo y parentesco en el Chaco Arido Serrano. Córdoba: Centro de Estudios Avanzados, Universidad Nacional de Córdoba.

IADIZA - Instituto Argentino de Investigaciones de las Zonas Áridas (2008). Informe Calidad del Agua. Mendoza.

Leff, Enrique (2004). Racionalidad ambiental. La reapropiación social de la naturaleza. México, D.F.: Siglo XXI.

Llambí, Luis (1981). Las unidades de producción campesinas en el sistema capitalista: un intento de teorización. Estudios Rurales Latinoamericanos, 2(2), 125-154.

Machado Aráoz, Horacio y Paz, Federico (2016). Extractivismo: metabolismo necroeconómico del capital y fagocitosis de las agro-culturas. Reflexiones y aprendizajes desde las re-existencias campesinas en el Valle del Conlara. En Carlos Walter Porto-Gonçalves y Luis Daniel Hocsman (coords.), Despojos y resistencias en América Latina / Abya Yala (pp. 141-168). Buenos Aires: Estudios Sociológicos.

Marx, Karl (2002). El capital. Buenos Aires: Siglo XXI. 
Narotzky, Susana (2004). Antropología económica. Nuevas tendencias. Barcelona: Melusina.

Pastor, Gabriela (2005). Patrimonio, vivienda y agua en el paisaje del noreste mendocino. Uso y gestión del agua en tierras secas, 11: El agua en Iberoamérica. Mendoza: Programa Iberoamericano de Ciencia y Tecnología para el Desarrollo (CYTED).

Polanyi, Karl (1974). El sistema económico como proceso institucionalizado. En M. Godelier (comp.), Antropología y economía. Barcelona: Anagrama.

Porto Gonçalves, Carlos Walter (2006). El desafío ambiental. México, D.F.: Programa de las Naciones Unidas para el Medio Ambiente (PNUMA-ONU).

Prada, Raúl (2014). Horizontes de los mundos posibles. ¿De qué se trata? Alter-nativa. Revista de Estudios Rurales, 1(1), 108-127.

Quintero Weir, José Ángel (2011). Wopukarü jatumi wataawai: el camino hacia nuestro propio saber. Reflexiones para la construcción autónoma de la educación indígena. Utopía y Praxis Latinoamericana, 16(54), 93-116. Universidad del Zulia, Maracaibo, Venezuela.

Torres, Laura (2008). Las racionalidades de unos y otros en el proceso de lucha contra la desertificación: el caso de los productores caprinos del noreste de Mendoza (tesis de maestría). Facultad de Filosofía y Humanidad, Universidad Nacional de Córdoba, Córdoba.

Torres, Laura (2010). Claroscuros del desarrollo sustentable y la lucha contra la desertificación: las racionalidades económicas en el ojo de la tormenta. Estudio de caso con productores caprinos de tierras secas (Mendoza, Argentina). Mundo Agrario, 11(21). Universidad Nacional de La Plata, La Plata.

Rivera Cusicanqui, Silvia (2010). Oprimidos pero no vencidos. Luchas del campesinado aymara y qhechwa 1900-1980. La Paz: La Mirada Salvaje.

Sahlins Marshall (1983). Economía de la edad de piedra. Madrid: Akal.

Schiavoni, Gabriela (1995). Colonos y ocupantes: parentesco, reciprocidad y diferenciación social en la frontera agraria de Misiones. Posadas: Universitaria.

Van der Ploeg, Jan Dow (2008). The new peasantries. Struggles for Autonomy and Sustainability in an Era of Empire and Globalization. London: Earthscan.

Weber, Max (2003). Economía y sociedad. Esbozo de sociología comprensiva. Madrid: FCE.

Weber, Max (2009). La ética protestante y el espíritu del capitalismo. Laeditorialvirtual. com.ar. Recuperado de https://fortunatti.files.wordpress.com/2015/04/la-eticaprotestante-y-el-espiritu-del-capitalismo.pdf. 